\title{
Construction of Exact Parametric or Closed Form Solutions of Some Unsolvable Classes of Nonlinear ODEs (Abel's Nonlinear ODEs of the First Kind and Relative Degenerate Equations)
}

\section{Dimitrios E. Panayotounakos and Theodoros I. Zarmpoutis}

Division of Mechanics, Department of Applied Mathematical and Physical Sciences, National Technical University of Athens, Zographou, 15773 Athens, Greece

Correspondence should be addressed to Theodoros I. Zarmpoutis, zarmpoutis@tee.gr

Received 10 May 2011; Revised 9 August 2011; Accepted 29 August 2011

Academic Editor: N. Govil

Copyright (C) 2011 D. E. Panayotounakos and T. I. Zarmpoutis. This is an open access article distributed under the Creative Commons Attribution License, which permits unrestricted use, distribution, and reproduction in any medium, provided the original work is properly cited.

\begin{abstract}
We provide a new mathematical technique leading to the construction of the exact parametric or closed form solutions of the classes of Abel's nonlinear differential equations (ODEs) of the first kind. These solutions are given implicitly in terms of Bessel functions of the first and the second kind (Neumann functions), as well as of the free member of the considered ODE; the parameter $v$ being introduced furnishes the order of the above Bessel functions and defines also the desired solutions of the considered ODE as one-parameter family of surfaces. The nonlinear initial or boundary value problems are also investigated. Finally, introducing a relative mathematical methodology, we construct the exact parametric or closed form solutions for several degenerate Abel's equation of the first kind.
\end{abstract}

\section{Introduction}

One of the most known nonlinear differential equations (ODEs) is the Abel equation of the first kind. There are admissible functional transformations that can reduce the general first kind nonlinear Abel (ODE) $\left(u_{\xi}^{\prime}=f_{3}(\xi) u^{3}+f_{2}(\xi) u^{2}+f_{1}(\xi) u+f_{0}(\xi)\right)$ to an Abel equation of the first kind of the normal form $\left(y_{x}^{\prime}=y^{3}+F(x)\right)$. None of these types of equations admits general solutions in terms of known (tabulated) functions, since only very special cases (depending on the form of the free member $F(x)$ or on $\left.f_{i}(\xi), i=0,1,2,3\right)$ can be solved in parametric form [1-3]. We underline here that adding a cubic term in a Riccati (ODE), an Abel nonlinear (ODE) of the first kind arises.

Our motivation of studying this problem was to provide a mathematical methodology through which the construction of exact parametric or closed form solutions of an Abel 
first kind nonlinear ODE form is possible. According to this method, separating properly the terms of the considered ODE, one leads to an equivalent system of two equations. The first one is a Riccati ODE, and the second one is a cubic equation that must be simultaneously hold true. In other words, any solution which satisfies these two equations (the Riccati ODE and the cubic one) satisfies also the Abel equation of the first kind of the normal form. Using basic results given by Kamke [1], the above Riccati equation is transformed to a second-order linear ODE of variable and determinable coefficients. We succeed in defining the coefficients of the above second-order linear ODE, so that the prescribed cubic equation hold true, and in the sequel in solving exactly this secondorder linear ODE. This introducing procedure guides to the construction of the abovementioned exact parametric or closed form solutions of the initial Abel equation. Here, we underline that all the above introduced functional transformations are admissible and that several basic results concerning the solutions of such types of equations are included in Zarmpoutis [4]. A nonlinear initial or boundary value problem is investigated under the assumption that the constructed solution of the Abel equation is unique inside a main interval of the independent variable. Finally, relative to the above mathematical methodology, we succeed in constructing the exact or closed form solutions for several degenerate Abel's nonlinear ODEs of the first kind special cases which are introduced in [5-7].

\section{Analysis-Construction of the General Solutions}

An Abel equation of the first kind has the general form

$$
u_{\xi}^{\prime}=f_{3}(\xi) u^{3}+f_{2}(\xi) u^{2}+f_{1}(\xi) u+f_{0}(\xi), \quad u=u(\xi)
$$

Here, the notation $y_{x}^{\prime}=d y / d x, y_{x x}^{\prime \prime}=d^{2} y / d x^{2}, \ldots$ is used for the total derivatives.

The admissible functional transformations [3, Chapter 0.1.5-2]:

$$
x=\int f_{3} E^{2} d \xi, \quad y=\left(u+\frac{f_{2}}{3 f_{3}}\right) E^{-1}, \quad E=\exp \left[\int\left(f_{1}-\frac{f_{2}^{2}}{3 f_{3}}\right) d x\right]
$$

brings the general form (2.1) in the canonical normal form:

$$
\begin{gathered}
y_{x}^{\prime}=y^{3}+F(x), \\
F(x)=\frac{1}{f_{3} E^{3}}\left[f_{0}+\frac{1}{3}\left(\frac{f_{2}}{f_{3}}\right)_{x}^{\prime}-\frac{f_{1} f_{2}}{3 f_{3}}+\frac{2 f_{2}{ }^{3}}{27 f_{3}{ }^{2}}\right] .
\end{gathered}
$$


The first step of our new mathematical method is to separate properly the terms of the Abel equation (2.3a) in order to find a more convenient system of equations. In more details, (2.3a) can be rewritten as

$$
\begin{gathered}
y_{x}^{\prime}=(y+f)\left(y^{2}-f y+f^{2}\right), \\
f=\sqrt[3]{F}
\end{gathered}
$$

such that the solution of (2.3a) identically satisfies the equation

$$
y_{x}^{\prime}+f\left(y^{2}-f^{2}\right)=(y+f) y^{2}
$$

Introducing a subsidiary, smooth function $Q(x)$, one splits (2.5) into the following two equivalent equations:

$$
\begin{gathered}
y_{x}^{\prime}+f y^{2}-f^{3}=Q(x), \\
(y+f) y^{2}=Q(x) .
\end{gathered}
$$

The first of them (2.6a) is a Riccati (ODE), while the second one (2.6b) is a cubic equation. In fact, the elimination of $Q(x)$ between (2.6a) and (2.6b) leads to (2.4a). This means that any solution of the Riccati ODE (2.6a), that satisfies the restriction (2.6b), is also a solution of the Abel equation (2.4a) and (2.4b). It is well known that any Riccati equation can be transformed to a second-order linear ODE of variable coefficients [1].

The second step of our method is to construct a solvable second-order linear ODE of variable coefficients, which is equivalent to the Riccati (2.6a) that satisfies the restriction (2.6b). A kind of solvable Riccati equation of the normal form [3, pp 105; type 38] is of the type

$$
y_{x}^{\prime}=\frac{f_{x}^{* \prime}}{g^{*}} y^{2}-\frac{g_{x}^{* \prime}}{f^{*}}
$$

which admits a particular solution of the form

$$
y_{p}=-\frac{g^{*}}{f^{*}}
$$

where $f^{*}$ and $g^{*}$ are arbitrary functions that must be determined. 
Supposing that the Riccati equation (2.6a) can be written in the form (2.7), in order to solve (2.6a), it is necessary to define the above-mentioned $f^{*}$ and $g^{*}$. Thus, one reads

$$
\begin{gathered}
f_{x}^{* \prime}=-f g^{*}, \\
f^{*}=-\frac{g_{x}^{* \prime}}{f^{3}+Q^{\prime}}
\end{gathered}
$$

so that (2.6a) admits a particular solution of the type (2.8).

From now on, differentiating $(2.9 \mathrm{~b})$, we extract

$$
g_{x x}^{\prime \prime *}=-\left(f^{3}+Q\right)_{x}^{\prime} f^{*}-\left(f^{3}+Q\right) f_{x}^{* \prime},
$$

while combining the last equation with (2.9a) and (2.9b), one gets

$$
g_{x x}^{\prime \prime *}-\frac{\left(f^{3}+Q\right)_{x}^{\prime}}{f^{3}+Q} g_{x}^{* \prime}-f\left(f^{3}+Q\right) g^{*}=0
$$

By now, the system (2.9a) and (2.9b) becomes

$$
\frac{g_{x x}^{* \prime \prime}}{f g^{*}}-\frac{\left(f^{3}+Q\right)_{x}^{\prime}}{\left(f^{3}+Q\right) f}-\frac{g_{x}^{* \prime}}{g^{*}}=\left(f^{3}+Q\right), \quad f^{*}=-\frac{g_{x}^{* \prime}}{f^{3}+Q} .
$$

Introducing a second subsidiary differentiable function $\Omega(x)$ such that

$$
\Omega(x)=f^{3}+Q(x)=f^{3}+f y^{2}+y^{3},
$$

system (2.12) can be rewritten as

$$
\begin{gathered}
g_{x x}^{* \prime \prime}-\frac{\Omega_{x}^{\prime}}{\Omega} g_{x}^{* \prime}-f g^{*} \Omega=0, \\
f^{*}=\frac{g_{x}^{* \prime}}{\Omega} .
\end{gathered}
$$

According to [1, p 441; type 15], the Bessel second-order linear ODE

$$
z_{x x}^{\prime \prime}-\left[\frac{G_{x x}^{\prime \prime}}{G_{x}^{\prime}}+(2 \mu-1) \frac{G_{x}^{\prime}}{G}\right] z_{x}^{\prime}+\left[\left(\mu^{2}-v^{2}\right)\left(\frac{G_{x}^{\prime}}{G}\right)^{2}+G_{x}^{\prime 2}\right] z=0,
$$

where $G(x)$ is an arbitrary smooth function and $\mu, v$ are arbitrary parameters, admits the two partial linearly independent solutions $G^{\mu} J_{v}(G)$ and $G^{\mu} Y_{v}(G)$. Here, $J_{v}(G)$ and $Y_{v}(G)$ 
are the Bessel functions of the first and the second kind (Neumann function) of order $v$, respectively. Thus, the general solution of (2.15) is given by

$$
z=G^{\mu} Z_{v}(G)=G^{\mu}\left[C_{1} J_{v}(G)+C_{2} Y_{v}(G)\right], \quad C_{1}, C_{2}=\text { integration constants. }
$$

Comparing now (2.14a) with (2.15), one finds

$$
\begin{gathered}
\frac{G_{x x}^{\prime \prime}}{G_{x}^{\prime}}+(2 \mu-1) \frac{G_{x}^{\prime}}{G}=\frac{\Omega_{x}^{\prime}}{\Omega}, \\
\left(\mu^{2}-v^{2}\right)\left(\frac{G_{x}^{\prime}}{G}\right)^{2}+G^{\prime 2}=-f \Omega,
\end{gathered}
$$

with

$$
\Omega(x)=f^{3}+Q(x)=f^{3}+y^{3}+f y^{2} .
$$

Here, $y(x)$ is the general solution of the Riccati equation (2.6a) under the restriction (2.6b). Integration of (2.17a) furnishes

$$
G_{x}^{\prime}=C \Omega G^{1-2 \mu} \Longleftrightarrow \frac{G_{x}^{\prime}}{G}=C \Omega G^{-2 \mu}, \quad C=\text { integration constant, } G(x) \neq 0,
$$

while inserting the result (2.18) into the second equation (2.17b), one finds

$$
G^{2}=-\frac{f+\left(\mu^{2}-v^{2}\right) C^{2} \Omega G^{-4 \mu}}{C^{2} \Omega G^{-4 \mu}}
$$

By the assumption $\mu=0$, the Bessel equation (2.15) together with (2.14a), (2.13), (2.6b), and (2.18) becomes

$$
\begin{gathered}
z_{x x}^{\prime \prime}-\left[\frac{G_{x x}^{\prime \prime}}{G_{x}^{\prime}}-\frac{G_{x}^{\prime}}{G}\right] z_{x}^{\prime}+\left[G_{x}^{\prime 2}-v^{2}\left(\frac{G_{x}^{\prime}}{G}\right)^{2}\right] z=0, \\
g_{p_{1}}^{*}(x)=J_{v}(G), \quad g_{p_{2}}^{*}(x)=Y_{v}(G), \\
G^{2}(x)=\frac{v^{2} C^{2} \Omega-f}{C^{2} \Omega} .
\end{gathered}
$$

Here, $g_{p_{1}}^{*}, g_{p_{2}}^{*}$ are two partial linearly independent solutions of the Bessel (ODE) (2.14a). 
By now, using equations (2.9b), (2.8), and (2.18), the restriction (2.6b) as well as the properties of the Bessel functions $[3,8,9]$, we get the estimation

$$
f_{p_{1}}^{*}=-\frac{\left(g_{p_{1}}^{*}\right)_{x}^{\prime}}{\Omega}=-\frac{1}{C}\left[J_{v-1}(G)-J_{v+1}(G)\right], \quad f_{p_{2}}^{*}=-\frac{\left(g_{p_{2}}^{*}\right)_{x}^{\prime}}{\Omega}=-\frac{1}{C}\left[Y_{v-1}(G)-Y_{v+1}(G)\right]
$$

$$
G^{2}(x)=\text { as in equation (2.19) for } \mu=0 \text {. }
$$

Thus, the two partial linearly independent solutions of the Riccati equation (2.6a) $y_{p_{1}}$, $y_{p_{2}}$ under the restriction $(2.6 \mathrm{~b})$ become

$$
y_{p_{1}}(x)=\frac{2}{C} \frac{J_{v}(G)}{J_{v-1}(G)-J_{v+1}(G)}, \quad y_{p_{2}}(x)=\frac{2}{C} \frac{Y_{v}(G)}{Y_{v-1}(G)-Y_{v+1}(G)},
$$

where

$$
G^{2}(x)=\frac{v^{2} C^{2} \Omega-f}{C^{2} \Omega}, \quad \Omega(x)=f^{3}+y^{3}+f y^{2} .
$$

Furthermore, according to [3], the general solution of the above Riccati equation (2.6a) under the restriction $(2.6 b)$ is given by the formula

$$
\begin{gathered}
y=\frac{C y_{p_{1}}+U y_{p_{2}}}{C+U}, \quad U=\exp \left[\int f\left(y_{p_{1}}-y_{p_{2}}\right) d x\right] \\
C=\text { integration constant. }
\end{gathered}
$$

Solving the first of them for $U$ and taking the derivatives in both (2.21a) and (2.21b), we obtain the result

$$
\left(y_{p_{2}}-y_{p_{1}}\right)\left(y-y_{p_{1}}\right)_{x}^{\prime}-\left(y_{p_{2}}-y_{p_{1}}\right)_{x}^{\prime}\left(y-y_{p_{1}}\right)=f\left(y-y_{p_{1}}\right)\left(y_{p_{2}}-y_{p_{1}}\right)^{2} .
$$

Since any solution of the Riccati equation (2.6a) under the restriction (2.6b) is also a solution of the Abel equation (2.3a), we conclude that the general solution (2.24a) of the Riccati (2.6a) under the restriction (2.6b) is also the general solution of the Abel equation (2.3a). Taking into account the relations

$$
y_{x}^{\prime}=y^{3}+f^{3}, \quad\left(y_{p_{1}}\right)_{x}^{\prime}=y_{p_{1}}^{3}+f^{3}, \quad\left(y_{p_{2}}\right)_{x}^{\prime}=y_{p_{2}}^{3}+f^{3},
$$

we derive that the general solution of the Abel nonlinear ODE (2.4a) or (2.3a) and (2.3b) is given by the implicit functional relation

$$
\left(y-y_{p_{1}}\right)\left(y+y_{p_{1}}+y_{p_{2}}\right)=f\left(y_{p_{1}}-y_{p_{2}}\right)
$$


or, equivalently, the implicit functional relation

$$
\begin{gathered}
\left(\frac{2}{G} y-\frac{Y_{v}(G)}{Y_{v-1}(G)-Y_{v+1}(G)}\right)\left(\frac{2}{G} y+\frac{Y_{v}(G)}{Y_{v-1}(G)-Y_{v+1}(G)}+\frac{J_{v}(G)}{J_{v-1}(G)-J_{v+1}(G)}\right) \\
=f\left(\frac{J_{v}(G)}{J_{v-1}(G)-J_{v+1}(G)}-\frac{Y_{v}(G)}{Y_{v-1}(G)-Y_{v+1}(G)}\right), \\
G^{2}(x)=v^{2}-\frac{f}{C^{2} \Omega}, \quad \Omega(x)=f^{3}+y^{3}+f y^{2}, \quad f=\sqrt[3]{F},
\end{gathered}
$$

where $F(x)$ is the free member of the considered Abel equation, while $C$ is an integration constant and $v$ is a free parameter denoting the order of Bessel functions being introduced.

From the above analysis, one concludes that the implicit functional relation (2.28) expressing the general solution of the Abel equation of the first kind of the normal form (2.3a) may not be unique inside a main interval of the values $\left[x^{0}, x^{1}\right)$ of the independent variable $x$, because of various types that function $G(x)$ (2.29) admits; in other words, it can be divided into several branches of solutions valid inside consecutive subintervals. In this case, matching of the corresponding solutions including various integration constants $C$ as well as various values of the parameter $v$ must be followed in all the consecutive subintervals that solution (2.28) changes.

\section{Nonlinear Initial or Boundary Value Problem}

We suppose that solution (2.28) remains unchanged inside the first main interval $\left[x^{0}, x^{1}\right)$. Following the initial data $x_{1}=x_{1}^{0}=0, y_{1}=y_{1}^{0}$, we provide by (2.29)

$$
G_{1}^{0^{2}}(x)=v_{1}^{0^{2}}-\frac{f}{C_{1}^{0^{2}} \Omega_{1}^{0}}, \quad \Omega_{1}^{0}=f^{0^{3}}+y_{1}^{0^{3}}+f^{0} y_{1}^{0^{2}}, \quad x=x^{0}=0, \quad y=y^{0}
$$

where the first upper right index denotes the value of the corresponding quantity at the origin $x^{0}=0, y=y^{0}$. Thus, for the origin $\left(0, y_{1}^{0}\right)$, we dispose the following nonlinear algebraictranscendental system of equations

$$
\begin{aligned}
& \text { Equation }(2.28) \Longrightarrow\left(\frac{2}{G_{1}^{0}} y_{1}^{0}-A^{0}\right)\left(\frac{2}{G_{1}^{0}} y_{1}^{0}+B^{0}\right)-f_{1}^{0} \Delta^{0}=0 \\
& \text { Derivative of }(2.28) \Longrightarrow\left[\left(\frac{2}{G}\right)_{x}^{\prime} y^{0}+\left(\frac{2}{G}\right)\left(y_{x}^{\prime}\right)^{0}-\left(A_{x}^{\prime}\right)^{0}\right] \\
& \cdot\left[\left(\frac{2}{G}\right)_{x}^{\prime} y^{0}+\left(\frac{2}{G}\right)\left(y_{x}^{\prime}\right)^{0}-\left(B_{x}^{\prime}\right)^{0}\right]=\left(f_{x}^{\prime}\right)^{0} \Delta+f\left(\Delta_{x}^{\prime}\right)^{0},
\end{aligned}
$$


where

$$
\begin{gathered}
G^{2}(x)=v^{2}-\frac{f}{C^{2} \Omega}, \quad G_{x}^{\prime}=\frac{1}{2 G}\left(1-\frac{1}{C^{2}} \frac{\Omega f_{x}^{\prime}+\Omega_{x}^{\prime} f}{\Omega^{2}}\right), \\
\Omega_{x}^{\prime}=3 f^{2} f_{x}^{\prime}+\left(3 y^{2}+2 f y\right) y_{x}^{\prime}, \quad y_{x}^{\prime}=y^{2}+f^{3}, \\
A_{x}^{\prime}=\left(\frac{Y_{v}}{Y_{v-1}-Y_{v+1}}\right)_{G}^{\prime} G_{x}^{\prime}, \quad B_{x}^{\prime}=\left(\frac{Y_{v}}{Y_{v-1}-Y_{v+1}}+\frac{J_{v}}{J_{v-1}-J_{v+1}}\right)_{G}^{\prime} G_{x}^{\prime} \\
\Delta_{x}^{\prime}=\left(\frac{J_{v}}{J_{v-1}-J_{v+1}}-\frac{Y_{v}}{Y_{v-1}-Y_{v+1}}\right) G_{x}^{\prime}, \\
J_{v}=\sum_{k=0}^{\infty} \frac{(-1)^{p}(G / 2)^{v+2 p}}{k ! \Gamma(v+p+1)}, \quad Y_{v}=\frac{J_{v} \cos \pi v-J_{-v}}{\sin \pi v}, \\
J_{v_{G}}^{\prime}=\frac{1}{2}\left(J_{v-1}-J_{v+1}\right), \quad Y_{v_{G}}^{\prime}=\frac{1}{2}\left(Y_{v-1}-Y_{v+1}\right) .
\end{gathered}
$$

The set of equations (3.2a) and (3.2b) is sufficient for the estimation of the constant of integration $C^{0}$ as well as for the parameter $v^{0}$, so that the one-parameter family of surfaces solution of the original Abel equation takes the concrete form with given $v=v^{0}$ and $C=C^{0}$.

\section{Applications and Discussion}

In this section, we will examine some special cases of degenerate Abel's nonlinear ODEs of the first kind that cover all the examined applications [5-7]. A modified mathematical methodology is introduced that simplify the mathematical methodology and technique being introduced in previous sections. We will investigate and discuss two degenerate type of equations, that is,

$$
y_{x}^{\prime}=f(x) y^{3}+g(x) y^{2}+h(x) \quad \text { or } y_{x}^{\prime}=f(x) y^{3}+g(x) y^{2} .
$$
setting

Consider the first of these equations (introducing $h(x)=0$ ). From the second of these,

$$
y(x)=\frac{1}{u(x)}, \quad u(x) \neq 0,
$$

we extract

$$
u u_{x}^{\prime}=f_{1}(x) u^{2}+f_{2}(x) u+f_{3}(x)
$$

which, further, using the functional transformation [3, page 50]

$$
w(x)=u(x) E(x), \quad E(x)=\exp \left(-\int f_{1}(x) d x\right),
$$


results in the simpler form

$$
w w_{x}^{\prime}=F_{1}(x) w+F_{0}(x), \quad F_{1}(x)=f_{2}(x) E(x), \quad F_{0}(x)=f_{3}(x) E^{2}(x) .
$$

This new equation is an Abel equation of the second kind that, further, by the substitution [3, page 40]

$$
\xi(x)=\int F_{1}(x) d x=\int f_{2}(x) \exp \left(-\int f_{1} d x\right) d x=\Omega(x) \Longleftrightarrow x=\Omega^{-1}(\xi),
$$

becomes an Abel equation of the second kind of the normal form, that is,

$$
y(\xi) y_{\xi}^{\prime}(\xi)-y(\xi)=\mathcal{F}(\xi), \quad \mathcal{F}(\xi)=\frac{F_{0}(\xi)}{F_{1}(\xi)}=\frac{F_{0}\left(\Omega^{-1}(\xi)\right)}{F_{1}\left(\Omega^{-1}(\xi)\right)}
$$

This last equation by the admissible substitution

$$
y(\xi)=\frac{1}{\omega(\xi)}, \quad \omega(\xi) \neq 0, \quad y_{\xi}^{\prime}=-\frac{\omega_{\xi}^{\prime}}{\omega}
$$

reduces to the following Abel degenerate nonlinear ODE of the first kind:

$$
\omega_{\xi}^{\prime}=-\mathscr{F}(\xi) \omega^{3}+\omega^{2}
$$

Using the final admissible functional transformation [1]

$$
\omega(\xi)=-\frac{1}{t \xi_{t}^{\prime}(t)}, \quad t \xi_{t}^{\prime} \neq 0
$$

performing

$$
w_{\xi}^{\prime}=-\left(\frac{1}{t \xi_{t}^{\prime}}\right) t_{\xi}^{\prime}=\frac{\xi^{\prime}-t \xi_{t t}^{\prime \prime}}{t^{2} \xi_{t}^{\prime 2}} \frac{1}{\xi_{t}^{\prime}}=\frac{1}{t^{2} \xi_{t}^{\prime 2}}-\frac{\xi_{t t}^{\prime \prime}}{t \xi_{t}^{\prime 3}}
$$

we transform (4.9) to the new nonlinear ODE of the second kind of the Emden-Fowler type, namely,

$$
\xi_{t t}^{\prime \prime}=-t^{-2} \mp(\xi) ; \quad t \neq 0,
$$

where $\mathcal{F}(\xi)$ is a known smooth function of the variable $\xi$. 
By now, one uses the mathematical methodology and the results are included in [10]. In fact, introducing the functional transformation

$$
\begin{gathered}
u=t^{-2} \Longrightarrow d u=2 t^{-3} d t=2 u \sqrt{u} d t \\
z=t^{-2} \frac{\xi_{t}^{\prime}}{\xi} \Longrightarrow d z=\left[\frac{t^{-2}}{\xi} \xi_{t t}^{\prime \prime}+\left(\frac{t^{-2}}{\xi}\right)_{t}^{\prime}\right] d t
\end{gathered}
$$

one calculates the total differentials $d u$ and $d z$ and finally derives

$$
u_{z}^{\prime}=\frac{d u}{d z}=\frac{u \sqrt{u}}{(\mp(\xi) / \xi) u^{2}-2 z \sqrt{u}-z^{2} u} .
$$

Furthermore, setting

$$
\sqrt{u(t)}=P(t)
$$

and supposing that $G(z) \equiv\left(\mathcal{F}(\xi) P^{3} / \xi\right)-2 z P$, we derive the following nonlinear ODE when $G(z)$ is a function which must be determined:

$$
P_{z}^{\prime}=\frac{P}{G(z)-z^{2} P} ; \quad G \equiv G(z)=\frac{\mathcal{F}(\xi)}{\xi} P^{3}-2 z
$$

Thus, the ODE (4.14) becomes the following first-order Abel's nonlinear type of equations

$$
\left(-z^{2} P+G\right) P_{z}^{\prime}=P ; \quad P=P(z)
$$

We apply now the Julia construction [1, page 27], and we derive the following functional relation between its coefficients:

$$
-2 z G=-z^{2}\left(1+G_{z}^{\prime}\right)
$$

namely, the linear first-order ODE

$$
z G_{z}^{\prime}-2 G+z=0
$$

with general solution given by

$$
G(z)=z+\lambda z^{2}
$$


where $\lambda$ is a free parametric (constant of integration). Then, the general solution of the Abel nonlinear ODE (4.17), according to the results [1, page 27], becomes

$$
P^{2}-\frac{2+\lambda z}{z} P=\mu,
$$

where $\mu$ is another free parameter (constant of integration). Solving the last equation for $P$, one finally derives

$$
P=u^{1 / 2}=\frac{2+\lambda z}{z} \pm \sqrt{\left(\frac{2+\lambda z}{z}\right)^{2}-\mu,} \quad z \text { =parameter }
$$

which, because of (4.15) and (4.13), defines the independent variable $t$ by the formula

$$
\begin{gathered}
t=\frac{1}{\sqrt{u}}=\frac{z}{(2+\lambda z) \pm \sqrt{(2+\lambda z)^{2}-\mu z}} ;-\infty<z<+\infty \\
\lambda, \mu=\text { free parameters (constants of integration). }
\end{gathered}
$$

Furthermore, the dependent variable $\xi$ can be estimated by the combination of (4.20) together with the second of (4.16), namely,

$$
\frac{\mp(\xi)}{\xi} u^{3 / 2}-2 z=z+\lambda z^{2},
$$

or equivalently

$$
\mathcal{F}(\xi)\left[\frac{(2+\lambda z) \pm \sqrt{(2+\lambda z)^{2}-\mu z^{2}}}{z}\right]^{3}-2 z=z+\lambda z^{2}
$$

by means of which one gets

$$
\begin{gathered}
\frac{\mathcal{F}(\xi)}{\xi}=\frac{(3+\lambda z) z^{4}}{\left[(2+\lambda z) \pm \sqrt{2+\lambda z-\mu z^{2}}\right]^{3}}, \quad-\infty<z<+\infty, \\
\mathcal{F}(\xi)=\text { known, } \lambda, \mu=\text { free parameters (constants of integrations). }
\end{gathered}
$$

Both (4.23) and (4.26) constitute the exact parametric solutions of the Emden-Fowler type nonlinear ODE (4.12).

For $\lambda=\mu=0$ (free parameters), the already constructed parametric solution becomes

$$
t=\frac{1}{4} z ; \quad \frac{\mathcal{F}(\xi)}{\xi}=\frac{3}{64} z^{4} \Longleftrightarrow \frac{\mathcal{F}(\xi)}{\xi}=12 t^{4},
$$


that, because $\mathcal{F}(\xi)$ is a known function of $\xi$, furnishes $\xi$ as function of $t$. From now on, one follows the inverse course. becomes

For $\lambda=0$ ( free parameter), the obtained parametric solutions (4.23) and (4.26)

$$
t=\frac{2}{2 \pm \sqrt{2-\mu z^{2}}}, \quad \frac{\mathcal{F}(\xi)}{\xi}=\frac{3 z^{4}}{(2 \pm \sqrt{2-\mu z})^{3}}
$$

that is,

$$
\frac{\mp(\xi)}{\xi}=3 t^{3} z= \pm 6 t^{3} \sqrt{\frac{t}{(1-2 \mu t)^{2}}}, \quad \mu=\text { integration constant. }
$$

Since $\mathcal{F}(\xi)$ is a known function of $\xi$, the last equation permits us to evaluate $\xi$ with respect to the independent variable $t$ and thus functions $w(\xi)$ and $y(\xi)$ through relations (4.10) and (4.8), respectively. Following the inverse course, one finally defines $y(x)$ by the way of (4.1).

Summarizing, we underline that, in this section, by using the results of [10], we proposed a new mathematical methodology and we succeed in giving new mathematical techniques in constructing analytic or closed form solutions of a class of degenerate Abel's ODEs in mathematical physics and nonlinear mechanics. The methodology being introduced simplifies that of Section 2, which refers to the solution of an Abel equation of the first kind. For strengthening, we refer to some categories of degenerate Abel's ODEs of the first kind which are included in [5-7], namely, (i) $(a x+b)^{2} y_{x}^{\prime}+(a x+b) y^{3}+c y^{3}=0$; (ii) $y_{x}^{\prime}+c y^{2}=0$; (iii) $x^{2} y_{x}^{\prime}+a y^{3}-a x^{2} y^{2}=0$; (iv) $y_{x}^{\prime}+\left(3 a x^{2}+4 x+b\right) y^{3}+3 x y^{2}=0$, and so forth.

\section{Conclusion}

A new mathematical technique leading to the construction of the exact parametric or closed form solutions of the classes of Abel's nonlinear differential equations (ODEs) of the first kind is presented. The solutions are given implicitly using Bessel's functions of the first and second kind (Neumann functions), the free member of the considered ODE as well as the subindex $v$, that defines the order of the Bessel functions. The nonlinear initial or boundary value problems are also investigated. Finally, through a new simpler mathematical methodology, we succeed in giving the exact parametric solutions of some degenerate cases of Abel's equation of the first kind included and investigated in $[1,5,7]$.

\section{References}

[1] E. Kamke, Differentialgleichungen, Lösungsmethoden und Lösungen. I: Gewöhnliche Differentialgleichungen, Neunte Auflage, Mit einem Vorwort von Detlef Kamk, B. G. Teubner, Stuttgart, Germany, 1977.

[2] G. M. Murphy, Ordinary Differential Equations and Their Solutions, D. Van Nostrand Co, Princeton, NJ, USA, 1960.

[3] A. D. Polyanin and V. F. Zaitsev, Handbook of Exact Solutions for Ordinary Differential Equations, Chapman \& Hall/CRC, Boca Raton, Fla, USA, 2nd edition, 2003.

[4] Th. I. Zarmpoutis, On the Construction of General Solutions of the Nonlinear ODEs Abel, Riccati and Emden- Fowler. Applications in nonlinear Mechanics and Mathematical physics, Ph.D. thesis, National Technical University of Athens, 2010. 
[5] E. S. Cheb-Terrab and A. D. Roche, "Abel ODEs: equivalence and integrable classes," Computer Physics Communications, vol. 130, no. 1, pp. 204-231, 2000.

[6] F. Schwarz, "Symmetry analysis of Abel's equation," Studies in Applied Mathematics, vol. 100, no. 3, pp. 269-294, 1998.

[7] M. P. Markakis, "Closed-form solutions of certain Abel equations of the first kind," Applied Mathematics Letters, vol. 22, no. 9, pp. 1401-1405, 2009.

[8] I. S. Gradsteyn and I. M. Ryzhik, Table of Integrals, Series and Products, Academic Press, New York, NY, USA, 5th edition, 1965.

[9] A. D. Polyanin and A. V. Manzhirov, Handbook of Mathematics for Engineers and Scientists, Chapman \& Hall/CRC, Boca Raton, Fla, USA, 2007.

[10] D. E. Panayotounakos, T. I. Zarmpoutis, and C. I. Siettos, "On the construction of the exact analytic or parametric closed form solutions of standing waves concerning the cubic Schrödinger equation," Archive of Applied Mechanics. In press. 


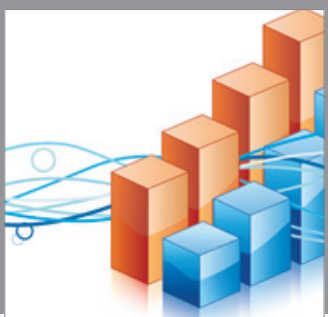

Advances in

Operations Research

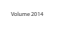

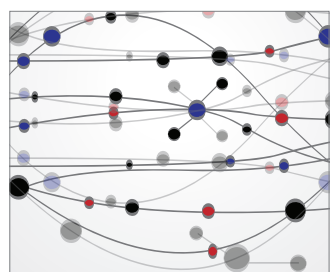

\section{The Scientific} World Journal
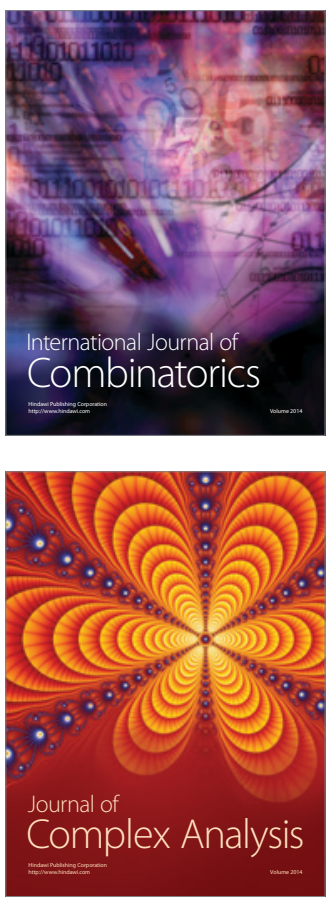

International Journal of

Mathematics and

Mathematical

Sciences
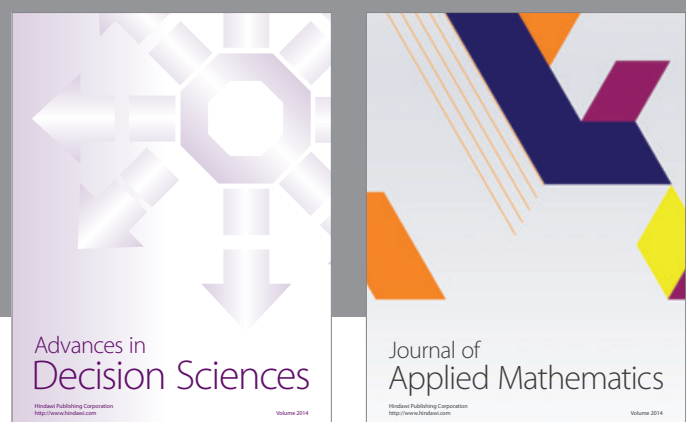

Journal of

Applied Mathematics
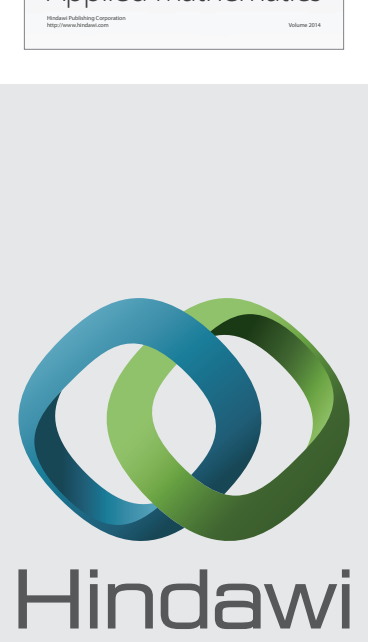

Submit your manuscripts at http://www.hindawi.com
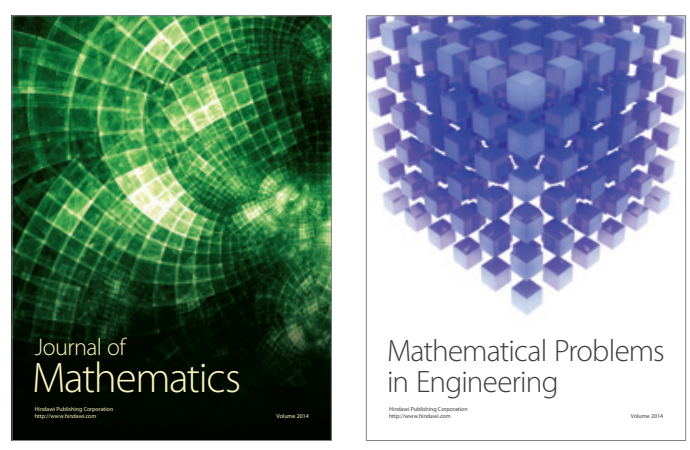

Mathematical Problems in Engineering
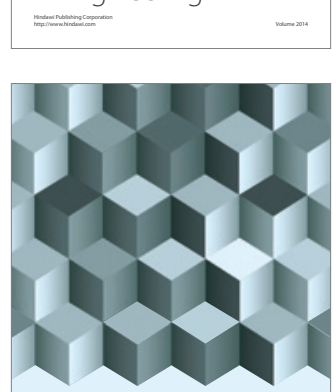

Journal of

Function Spaces
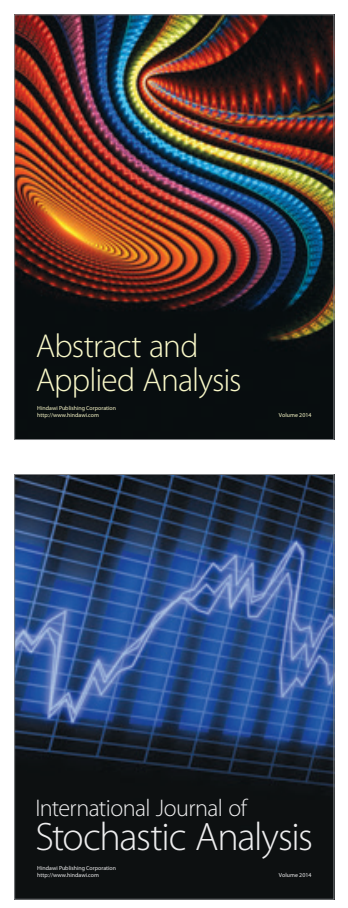

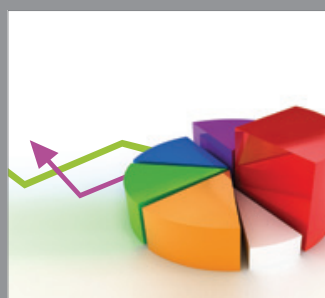

ournal of

Probability and Statistics

Promensencen
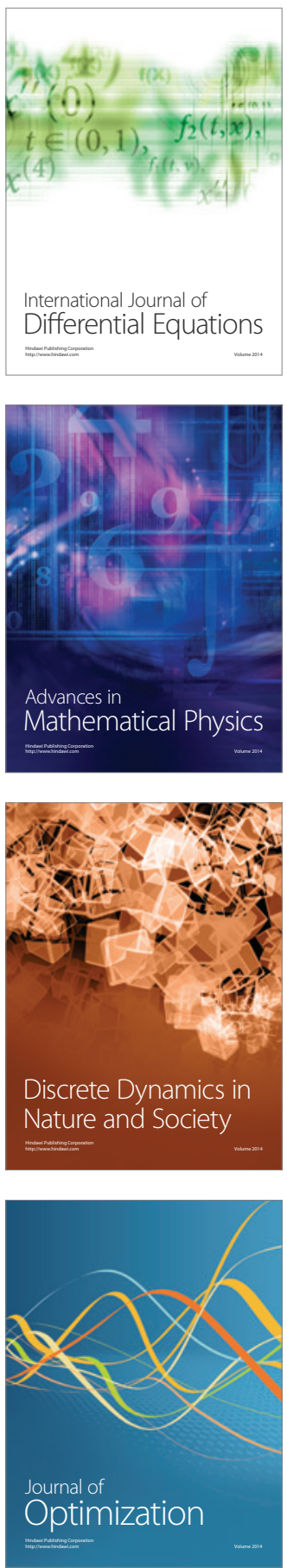